\title{
AVALIAÇÃO DO MODELO WRF PARA A PREVISÃO DO PERFIL DO VENTO NO CENTRO DE LANÇAMENTO DE ALCÂNTARA
}

\author{
ADAIANA F. GOMES DA SILVA ${ }^{1}$, GILBERTO FISCH ${ }^{2}$ \\ ${ }^{1}$ Centro de Previsão do Tempo e Estudos Climáticos/Instituto Nacional de Pesquisas Espaciais (CPTEC/INPE), \\ São José dos Campos, SP, Brasil \\ ${ }^{2}$ Instituto de Aeronáutica e Espaço (IAE), São José dos Campos, SP, Brasil \\ adaiana1@yahoo.com.br, fisch.gilberto@gmail.com
}

\begin{abstract}
RESUMO
Foi avaliada a capacidade do modelo WRF-ARW (versão 3.2.1) em prever o vento na região do Centro de Lançamento de Alcântara, visando aplicá-lo operacionalmente em ocasiões de lançamentos naquela base. Foram realizadas análises sazonais ao comportamento do modelo a partir de dois conjuntos de dados de radiossondagens representativos das estações seca (vento forte) e chuvosa (vento fraco). Previsões de 72 horas foram feitas a partir de condições iniciais fornecidas pelo Global Forecasting System (GFS/NCEP). Simulações configuradas para três domínios aninhados, resolução horizontal máxima de $1 \mathrm{~km}$ e vertical de 42 níveis foram comparadas com observações a cada 6 horas através do índice de concordância de Willmott (d). Foram realizados testes iniciais de sensibilidade para comparação e ajuste de diferentes parâmetros dinâmicos e físicos, como tamanhos de domínios, número de níveis verticais, spin-up time e parametrizações de Camada Limite Planetária. O modelo se mostrou razoável para representar o perfil vertical do vento, dentro de suas limitações, não mostrando diferença em seu desempenho entre as estações seca ou chuvosa e alcançando valores máximos de d na ordem de 0,90. Em geral, o modelo superestimou as componentes do vento ( $\mathrm{U}$ e $\mathrm{V}$ ) médias na camada em até $3,0 \mathrm{~m} / \mathrm{s}$ com relação às observações.
\end{abstract}

Palavras-chave: Engenharia do Vento, radiossondagem, simulação numérica, lançamento de foguetes.

\begin{abstract}
WRF MODEL ASSESSMENT FOR THE WIND PROFILE FORECAST AT THE ALCANTARA LAUNCHING CENTER

We evaluated the performance of the WRF-ARW model (version 3.2.1) to predict the winds at the Alcantara Launch Center applying it operationally on rocket launching occasions. Seasonal analysis of the model behavior were performed against two radiosonde data sets, which represent the dry (strong winds) and rainy (weak winds) seasons. 72 hours forecasts were made from initial conditions provided by the Global Forecasting System (GFS/NCEP). Simulations configured for three nested grid domains, maximum $1 \mathrm{~km}$ horizontal resolution and 42 vertical levels were compared with observations at each 6 hours through the Willmott index of agreement (d). Initial tests of sensitivity were made for comparison and adjustment of different physical and dynamic parameters, like size of domains, number of vertical levels, spin-up time and Planetary Boundary Layer parameterizations. The model was able to represent the vertical wind profile, showing no difference in performance between the dry or rainy seasons and reaching maximum values of $d$ in the order of 0.90 . Overall, the model overestimated the layer's average wind components ( $\mathrm{U}$ and $\mathrm{V}$ ) up to $3.0 \mathrm{~m} / \mathrm{s}$ with respect to the observations.
\end{abstract}

Keywords: Wind Engineering, radiosonde, numerical simulation, rocket launching. 


\section{INTRODUÇÃO}

O Centro de Lançamento de Alcântara (CLA) é considerado o "portal brasileiro para o espaço", sendo o local de lançamento de foguetes tais como o Veículo Lançador de Satélites (VLS) e foguetes de sondagem (VSB30, por exemplo), como parte integrante do Programa Espacial Brasileiro (AEB, 2012). O CLA está localizado na região nordeste brasileira, no norte do Estado do Maranhão, próximo de sua capital, São Luís (Figura 1). Esta é uma localização privilegiada para a construção de um centro de lançamento de foguetes devido à proximidade com a linha do Equador (latitude de $2^{\circ} \mathrm{S}$ ), faixa na qual o gasto de energia é bastante reduzido para colocação de satélites em órbita geoestacionária, possibilitando grande economia de combustível.

Nesta região equatorial, o regime de ventos é dominado pelos ventos alísios, que são fortes, persistentes e predominantemente de leste. Na literatura especializada são encontrados estudos específicos para o vento no CLA, dos quais citam-se alguns a seguir. Em termos de análises observacionais, Fisch (1999) realizou a primeira análise dos perfis de velocidade do vento, ventos máximos, fator, amplitude e amplitude máxima de rajadas e intensidade turbulenta do vento, considerando os períodos diurno e noturno para um período chuvoso e outro seco. Entre os resultados, estão que a turbulência é maior na estação chuvosa, que os ventos são mais intensos durante o dia na estação seca e durante a noite na estação chuvosa, e que existe uma rotação sazonal de nordeste (época chuvosa) para sudeste (época seca), em função do posicionamento da Zona de Convergência Intertropical (ZCIT). Ainda de acordo com este autor, durante a estação seca de Alcântara os ventos são mais fortes devido a uma superposição dos ventos alísios com a brisa marítima, que se torna mais intensa nessa época devido ao maior aquecimento da terra em contraste com as águas do oceano. Devido a este acoplamento, o vento se acelera ao penetrar o continente, porém é mais persistente e com um menor número de rajadas. Esta estação abrange os meses de julho a dezembro, sendo representativo o mês de setembro. $\mathrm{Na}$ estação chuvosa, o fenômeno de brisa é menos intenso devido ao menor contraste térmico e o vento é formado basicamente pelos alísios, por isso, no geral, é mais fraco. Esta estação vai de janeiro a junho, sendo mais representativos os meses de março e abril. Coutinho e Fisch (2007) compararam, através de métricas estatísticas, produtos oriundos de reanálise do modelo de circulação geral da atmosfera do European Center for Medium Range Weather Forecast (ECMWF) com saídas de radiossondagens realizadas no CLA e concluiram que o modelo simulou bem qualitativamente, mas superestimou os valores das radiossondagens, resultando erros de viés altos para o vento, da ordem de até $4,5 \mathrm{~m} / \mathrm{s}$. Roballo e Fisch (2008) estudaram parâmetros do escoamento atmosférico local a partir de dados de vento coletados pela torre anemométrica (TA) do CLA e de ensaios em túnel de vento, e observaram que o perfil logaritmico do vento é sazonal e que as velocidades estimadas pela lei de potência foram superestimadas comparadas às observadas durante a estação chuvosa, mas os valores foram equivalentes na estação seca, reforçando a hipótese da neutralidade atmosférica. Pires (2009), em seus estudos sobre a Camada Limite Interna (CLI) aplicados àquele local, utilizou os três pilares ideais para um estudo em micrometeorologia: observação com dados de torre e mastros anemométricos, ensaios em túnel de vento e um algoritmo computacional bidimensional das equações do movimento (Navier-Stokes) que foi utilizado para ajuste dos dados observacionais e linearização da equação do perfil

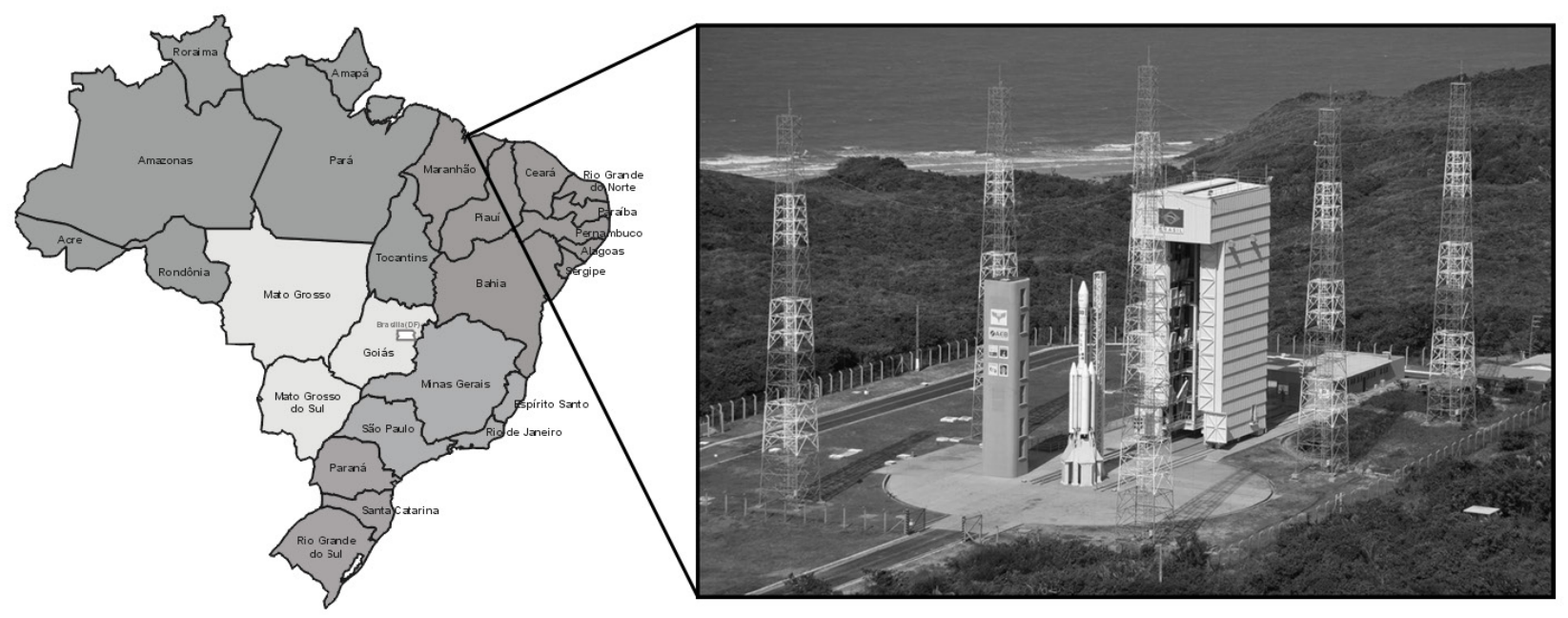

Figura 1 - Localização da área de estudo. 
logarítmico dos ventos. Foi constatado que a CLI atinge a altura de $17 \mathrm{~m}$ na torre móvel de integração e, como esta tem $20 \mathrm{~m}$ de altura, sofre forte influência da CLI, e que quanto mais alta é uma falésia, maior é a vorticidade, a altura da CLI e a extensão da bolha de recirculação, constituindo assim um fator importante na geração de turbulência a sotavento de falésias. Gisler (2009) fez uma análise estatística dos dados observados na TA do CLA e observou que mais de $93 \%$ das variâncias dos perfis de velocidade e direção do vento estiveram associadas aos sistemas atmosféricos atuantes, principalmente a ZCIT e a brisa marítima. Por último, Marciotto et al. (2012) caracterizaram os ventos de superfície a partir de uma série de dados medidos por aerovanes instalados a 1,5 e a $10 \mathrm{~m}$ de altura, ao longo de 10 dias representativos da estação seca da região, e propuseram adicionalmente uma fórmula heurística para estimar parâmetros de difusão, que podem vir a ser utilizados como entrada em modelos de dispersão.

As características do vento podem impactar direta e profundamente na trajetória de um foguete, podendo desviá-lo, causar imprevistos e até mesmo acidentes. Por este motivo é muito importante conhecer o regime de vento local em termos das características do perfil vertical. Não apenas seus padrões climatológicos, mas também suas condições exatas no momento do lançamento são cruciais para a segurança da operação. Podem-se verificar as condições meteorológicas através de medidas observacionais instantâneas imediatamente antes do lançamento, mas neste momento todo o equipamento já deve estar montado, checado e preparado. Um complemento para o prognóstico da determinação do vento é a previsão feita a partir de modelagem numérica. Observações realizadas com antecedência geram as condições iniciais necessárias para simular a dinâmica da atmosfera e representar o estado futuro a partir do tempo presente. Grandes avanços no desenvolvimento da modelagem numérica e dos próprios computadores possibilitaram a existência, atualmente, de diversos modelos globais e regionais, que são utilizados em inúmeras aplicações, sejam elas voltadas para a pesquisa ou operacionalmente para previsão do tempo e do clima. Os modelos regionais refinam a grade dos modelos globais $\left(10^{5} \mathrm{~m}\right)$ para meso $\left(10^{4} \mathrm{~m}\right.$ e $\left.10^{5} \mathrm{~s}\right)$ ou micro-escala $\left(10^{3} \mathrm{~m}\right.$ e $\left.10^{3} \mathrm{~s}\right)$. Isto permite simular grandes resoluções horizontais da ordem de até $10^{2} \mathrm{~m}$ e na vertical de até $10^{1} \mathrm{~m}$, sendo de segundos na escala temporal. Desta forma, através das condições iniciais e de contorno fornecidas pelos modelos globais, é possível captar as interações locais e ter uma melhor representação regional ou até mesmo pontual. Neste trabalho, o modelo atmosférico WRF (Weather Research and Forecasting), núcleo ARW (Advanced Research WRF), foi escolhido por se tratar do estado-da-arte em modelos numéricos (Wang et al., 2011; Carvalho et al., 2012). Além disso, é um software moderno, livre, de código aberto, fácil manipulação e com liberdade na configuração das simulações em termos de parâmetros numéricos, físicos e dinâmicos, o que permite grande adequação ao estudo específico. A importância da utilização deste modelo está também no desenvolvimento de uma autonomia operacional do Setor de Meteorologia do CLA para a realização de previsões locais às vésperas de uma operação de lançamento.

Assim, este trabalho teve o objetivo de avaliar a performance do modelo WRF na previsão do vento no CLA através da comparação com dados de radiossondagens para dois períodos distintos: um representativo de ventos mais fortes (período seco) e o outro de ventos mais fracos (período chuvoso), respectivamente setembro de 2008 e março de 2010. Também foi objetivada a determinação da melhor parametrização de Camada Limite Planetária (CLP) para o modelo WRF nas condições do CLA.

\section{DADOS E MÉTODOS}

\subsection{Dados observacionais}

Os dados observacionais foram obtidos em duas campanhas de radiossondagens realizadas no CLA, sendo uma representativa da estação seca (16 a 26/09/2008 Operação Murici II, IAE) e a outra da estação chuvosa (19 a 25/03/2010 - Projeto CHUVA-Alcântara GPM 2010, INPE). As radiossondagens foram realizadas nos quatro horários sinóticos diários (00:00, 06:00, 12:00 e 18:00 UTC) com o aparelho da marca Vaisala, modelo RS92-SVG.

O mês de março de 2010 foi marcado como atípico com relação à estação chuvosa da região. Chuvas intensas eram esperadas durante todo o mês, entretanto só iniciaram por volta do dia 19. Esta condição teve sua causa devida à ocorrência do fenômeno El-Niño nos primeiros meses de 2010. A Figura 2a mostra a série temporal da precipitação observada no mês de março de 2010, ao passo que a Figura $2 \mathrm{~b}$ apresenta a média mensal de chuvas para o período entre 1993 a 2008. Climatologicamente, o máximo de precipitação ocorre no mês de março e os mínimos em outubro e setembro, configurando os períodos utilizados para a presente análise como representativos das estações chuvosa e seca da região.

\subsection{Características das simulações}

O modelo WRF foi desenvolvido por um esforço conjunto de diversos órgãos americanos, entre eles o National Oceanic and Atmospheric Administration's (NOAA) e o National Center for Atmospheric Research (NCAR), que é operado pela University Corporation for Atmospheric Research (UCAR). Foi criado para fins de aplicação tanto em pesquisas como 

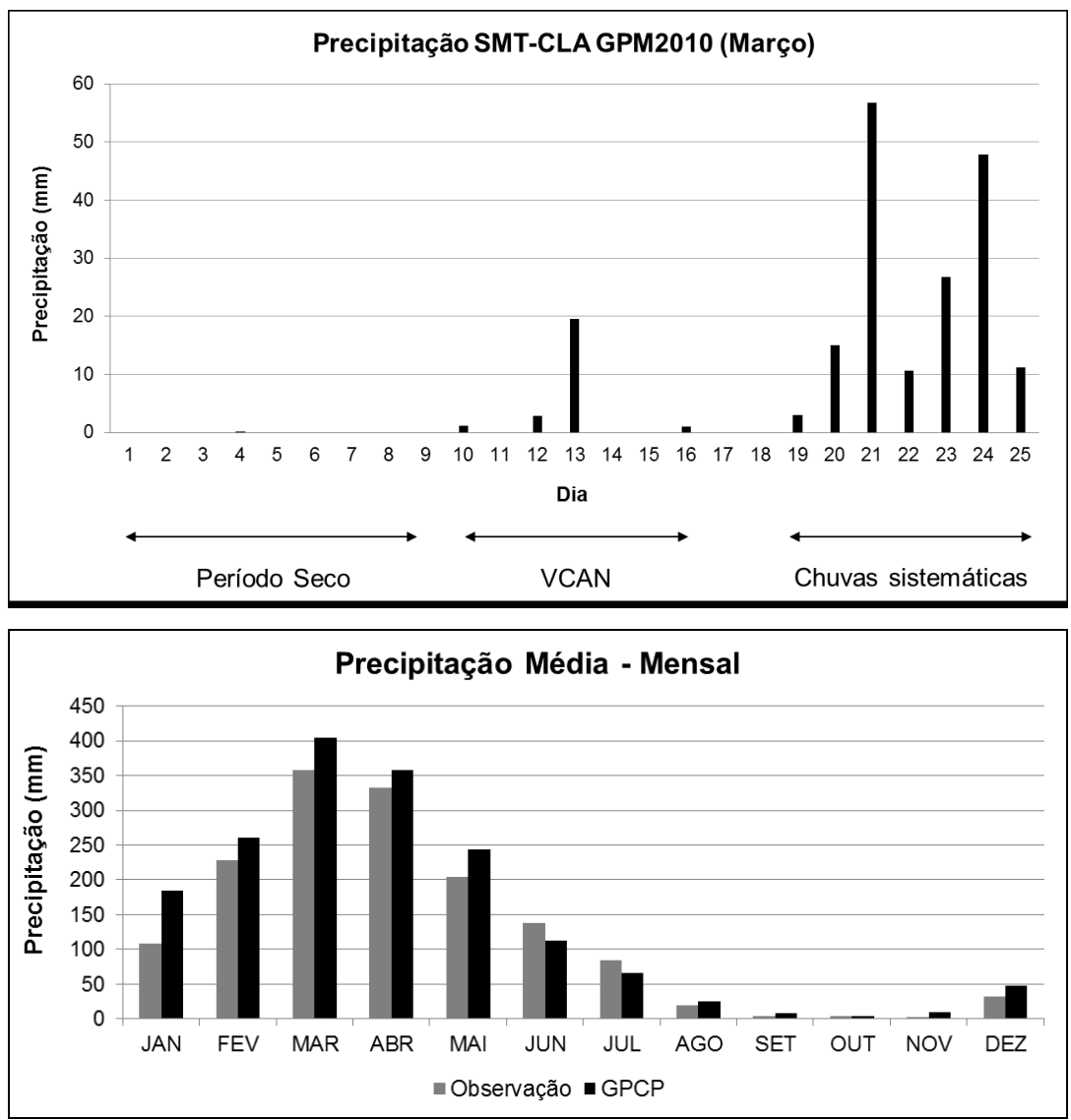

Figura 2 - (a) Série temporal da precipitação na região do CLA durante março de 2010. (b) Média mensal de precipitação para o CLA para o período entre 1993 a 2008, dados do Global Precipitation Climatology Project (GPCP).

Fonte: Relatório experimento Chuva GPM 2010. IAE, ICEA (2010).

também operacionalmente em Previsão Numérica do Tempo e é considerado o sucessor e uma evolução do modelo MM5.

As simulações numéricas são realizadas em quatro dimensões $(\mathrm{x}, \mathrm{y}, \mathrm{z}, \mathrm{t})$, pois a cada passo de tempo a malha horizontal repete-se em todos os níveis verticais. Os níveis verticais são fornecidos ao modelo em coordenadas sigma (Equação 1) que, junto ao solo, acompanham o contorno do terreno.

$$
\eta=\frac{p_{h}-p_{t o p}}{p_{s}-p_{t o p}}
$$

Para qual: $p_{\mathrm{h}}$ é a pressão em cada nível, $\mathrm{p}_{\text {top }}$ é a pressão no topo da atmosfera (constante) e $\mathrm{p}_{\mathrm{s}}$ é a pressão à superfície. $\eta$ varia de 0 a 1 , sendo 0 no topo da atmosfera e 1 na superfície.

A estrutura do modelo consiste de um pré-processamento realizado em um pacote de programas separados denominado WPS (WRF Preprocessing System), cuja saída é passada para o processamento principal (ARW Solver), primeiramente pelo programa "real" e, em seguida, para o WRF em si. Por fim, a saída final do WRF pode ser pós-processada para um formato amigável a programas de visualização gráfica, como o GrADS, por exemplo. Uma descrição ainda mais detalhada do modelo WRF pode ser encontrada em seu manual, em Skamarock et al. (2008) e também em Carvalho (2009).

Os dados utilizados para inicializar o modelo foram os campos gerados pela previsão do modelo global GFS, do National Centers for Environmental Prediction (NCEP), com resolução espacial de $0,5^{\circ} \times 0,5^{\circ}$ (aproximadamente $55 \times 55 \mathrm{~km}$ para a latitude local) e temporal de 6 horas, disponíveis em: http://nomads.ncep.noaa.gov/pub/data/nccf/ com $/ \mathrm{gfs} / \mathrm{prod} /$. A intenção de se utilizar dados de previsão e não de análise é para cumprir os objetivos de determinar a previsibilidade do modelo em condições possivelmente operacionais de uma campanha de lançamento de foguete, cujos resultados podem ser determinantes para o sucesso da missão, sendo parte integrante da motivação do trabalho.

Os dados do terreno foram fornecidos pelo United States Geological Survey (USGS) (disponíveis em: http://www.mmm. ucar.edu/wrf/src/wps_files/geog.tar.gz), com resolução espacial de 30" de arco. 
Dados de temperatura da superfície do mar (TSM) não foram inseridos por não se aplicarem a simulações curtas, devido à lenta variação da TSM. Na literatura, tal tarefa é recomendada apenas em casos de simulações longas (a partir de aproximadamente 10 dias). Uma análise da variação da TSM observada durante os períodos utilizados neste trabalho encontra-se detalhada em Silva (2013) e confirma a sua inexpressividade diante de uma simulação de 72 horas.

Um modelo com maior resolução vertical representa melhor a estrutura vertical da atmosfera, como mostram Nolan et al. (2009). Diversos outros trabalhos confirmaram também a melhoria na previsão usando grade horizontal mais refinada. Em Davis et al. (2010) é possível encontrar algumas citações a respeito. Ambas se relacionam na forma de que uma maior resolução horizontal exige maior resolução vertical. Contudo, apesar desta relação diretamente proporcional, existe um limite a partir do qual o incremento no número de níveis verticais não mais resulta em melhoria na qualidade da simulação.

Neste trabalho, todas as simulações foram realizadas com 42 níveis na vertical, três domínios quadrados e centralizados no

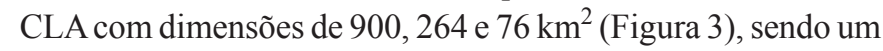
domínio mãe e dois aninhados, com comunicação bidirecional de dados entre eles. $\mathrm{O}$ espaçamento da grade do domínio maior foi de 9x9 km, reduzindo na proporção de 3 até a grade de 1x1 $\mathrm{km}$ no domínio menor, com passos de tempo de 45,15 e $5 \mathrm{~s}$ para os domínios 1, 2 e 3, respectivamente. Simulações com

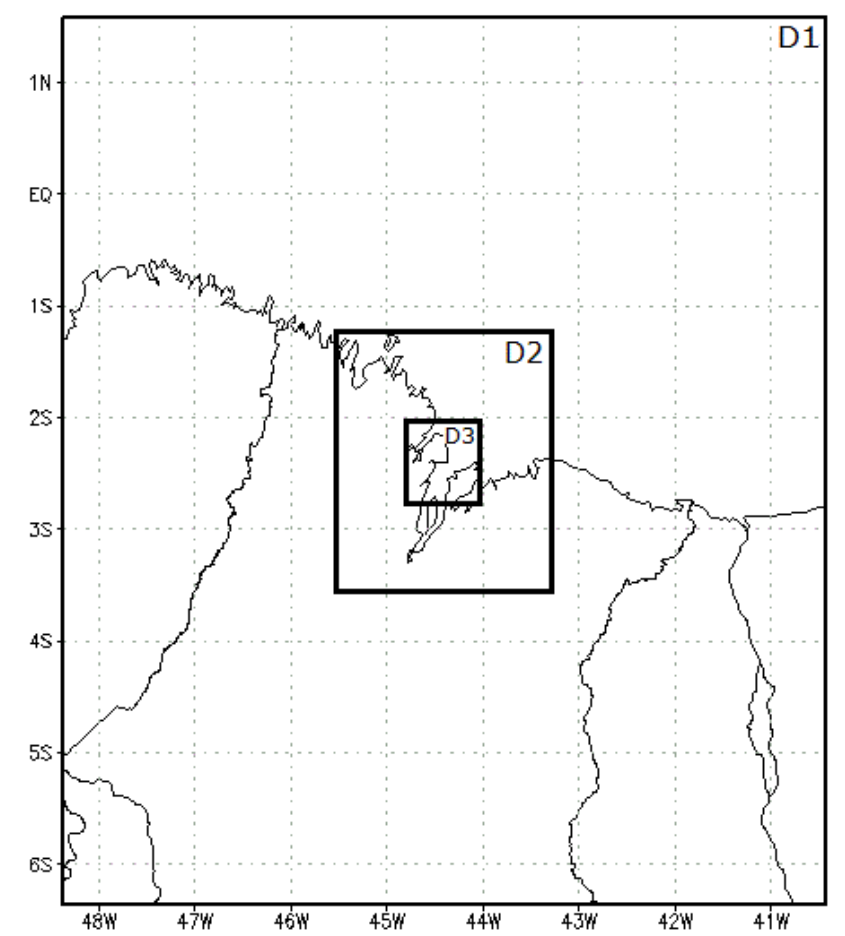

Figura 3 - Configuração de tamanhos dos domínios aninhados. tempo de integração de 72 horas, considerado o tempo inicial de spin-up de 6 horas, foram iniciadas a cada 12 horas (às $00 \mathrm{e}$ às 12 UTC) ao longo dos dias considerados, definidos na seção anterior. O número de níveis verticais, tamanho dos domínios e spin-up time adotados foram definidos por uma série de testes iniciais de sensibilidade para ajuste de parâmetros dinâmicos. Estes testes não serão abordados em detalhes neste texto, mas podem ser encontrados em Silva (2013).

Em geral, para avaliar quantitativamente a acurácia de modelos numéricos, utilizam-se métricas estatísticas para comparar dados observados e simulados. Para a metodologia deste trabalho, será utilizado o índice de concordância de Willmott (Willmott, 1981), cuja aplicação tem sido mais difundida e ampliada nos últimos anos (Emery et al., 2001; Wang et al., 2011).

Willmott (1981) parte da hipótese de que toda a variância de erros está contida na previsão e que a observação é livre de erros. Este índice especifica o grau no qual os desvios das observações em relação à média observada correspondem aos desvios das previsões em relação à média observada. É um índice adimensional limitado pelos valores 0 e 1 , onde 1 significa perfeita concordância entre observação e previsão e 0 significa completa discordância. Segundo Emery et al. (2001), o índice de Willmott condensa em uma única quantidade estatística todas as diferenças entre estimativas do modelo e observações dentro de uma dada região de análise e para um dado período de tempo.

O índice é calculado de acordo com a Equação 2 e será aplicado separadamente às componentes zonal (U) e meridional (V) do vento e para o vetor velocidade total (Vel), igualmente na fase de testes e na fase das simulações finais.

$$
d=1-\frac{\sum_{i=1}^{n}(P-O)^{2}}{\sum_{i=1}^{n}[|P-\bar{O}|+|O-\bar{O}|]^{2}}
$$

Para qual: $\mathrm{n}=$ número de níveis verticais, $\mathrm{P}=$ previsão, $\mathrm{O}=$ observação, $\bar{O}=$ média das observações, no caso, valor médio da camada.

\subsubsection{Testes de sensibilidade às parametrizações físicas de CLP}

Processos microfísicos, tais como radiação, nuvens Cumulus, trocas com a superfície, Camada Limite Planetária e convecção, têm cada um sua escala característica de ocorrência. Se o espaçamento da grade de simulação for maior, estes processos se tornam implícitos e precisam ser parametrizados. Existem diversos esquemas de parametrização disponíveis e cada um tem sua adequação de acordo com as particularidades da região e do caso a serem estudados, por isto muitos trabalhos têm sido realizados para testar a melhor escolha para o tipo 
de estudo que se pretende realizar. Entre diversos trabalhos, um exemplo de estudo recente é o de Carvalho et al. (2012), realizado para o modelo WRF.

Em todas as simulações deste trabalho, as parametrizações físicas utilizadas foram as default do WRF [Microfísica WRF Single Moment 3-class, Radiação RRTM para ondas longas e o esquema de Dudhia para ondas curtas, Camada superficial MM5 Similarity, Solo-superfície Noah-LSM, Cumulus Kain Fritsch nos domínios 1 e 2 e nenhum no domínio interno (explícito)].

Estas simulações de teste, também de 72 horas, iniciaram-se às 0000 UTC dos dias 20 e 21 de setembro de 2008 e de 20 e 21 de março de 2010, e testaram quatro diferentes tipos de parametrizações físicas de CLP (ACM2, MYJ, MYNN2,5 e YSU). Alguns detalhes de cada uma e as diferenças entre si encontram-se abaixo e a Tabela 1 resume as parametrizações de camada superficial e de interação solo-superfície utilizadas com cada esquema de CLP, de acordo com as recomendações do manual do modelo.

ACM2 (Asymmetric Convective Model version 2): esquema de fechamento de $1^{\mathrm{a}}$ ordem, sendo uma modificação do modelo convectivo de Blackadar. Possui ambas as componentes local e não-local para a mistura vertical descendente e ascendente, respectivamente.

MYJ (Mellor-Yamada-Janjic Scheme): operacional do modelo ETA, esquema de fechamento de energia cinética turbulenta (TKE), local. Possui uma equação prognóstica para a TKE.

MYNN2,5 (Mellor-Yamada Nakanishi and Niino Level 2,5): fechamento TKE, local, derivado do antigo esquema Burk-Thompson;

_ YSU (Yonsei University Scheme): esquema de fechamento de $1^{\text {a }}$ ordem, não-local, é uma evolução da parametrização do MRF, utilizada no modelo MM5. Estima a profundidade da camada de mistura.

\subsection{Padrão de Coerência Termodinâmica}

A ocorrência de precipitação pode alterar o escoamento do vento devido a processos associados à convecção e o escoamento das correntes descendentes associadas aos downdrafts (Gisler,
2009). O regime de chuvas pode influenciar o regime de ventos, e vice-versa. Por este motivo, particularmente para o período chuvoso, foi realizada adicionalmente uma análise de padrão de coerência termodinâmica, a fim de verificar o ciclo diário e a coincidência espaço-temporal das chuvas.

Foram utilizadas imagens geradas pelo Radar Banda $\mathrm{X}$ que esteve em operação no experimento Chuva Alcântara GPM 2010, com raios de alcance de 30 e $120 \mathrm{~km}$. As imagens são geradas a cada intervalo de 6 minutos e encontram-se disponíveis em: http://chuvaproject.cptec.inpe.br/portal/pedido. lista.logic.Estas foram comparadas com campos de precipitação acumulada de 1 hora e fração de nuvens baixas, geradas a partir de saídas horárias das simulações do WRF para o domínio interno de maior resolução. Para compensar a diferença entre os valores acumulados pelo modelo ao longo de 1 hora e os valores instantâneos de intensidade de precipitação do radar, todas as imagens do radar no intervalo entre determinada hora e sua antecedente foram utilizadas para a análise. Isto permitiu, de acréscimo, rastrear a trajetória de sistemas convectivos de escala maior, que se deslocavam sobre a área do domínio de simulação.

\section{RESULTADOS}

\subsection{Testes de sensibilidade}

Para comparar os resultados das simulações, foram construídas séries temporais do índice de Willmott calculado a cada 6 horas para as previsões de 72 horas iniciadas em 21/09/2008 e 21/03/2010 (figura não apresentada), sendo que na Figura 4 são apresentadas a média e o desvio-padrão (DP) destas séries temporais a fim de obter um valor que fosse representativo de cada simulação. Notou-se que, para o período seco, as linhas das diferentes parametrizações apresentaram um comportamento semelhante e, muitas vezes, chegaram a coincidir. Ao contrário do período chuvoso, cujas linhas foram divergentes, ou seja, os índices não foram semelhantes para as diferentes parametrizações. Na Figura 4, as setas indicam as mais altas médias e os mais baixos desvios-padrão de d. Comparando as quatro diferentes parametrizações entre

Tabela 1 - Resumo da distribuição de esquemas de parametrização física de camada limite.

\begin{tabular}{||c|c|c|c||}
\hline \hline \multicolumn{2}{|c|}{ CLP } & Camada superficial & Solo-Superfície \\
\hline ACM2 & $\begin{array}{c}\text { Fechamento 1 }{ }^{\text {a }} \text { ordem, local e } \\
\text { não-local }\end{array}$ & Pleim-Xiusurfacelayer & Pleim-Xiu LSM \\
\hline MYJ & Fechamento TKE, local & Similaridade ETA & Noah LSM \\
\hline MYNN 2,5 & Fechamento TKE, local & MYNN surfacelayer & Noah LSM \\
\hline YSU & ${\text { Fechamento 1 }{ }^{\text {a }} \text { ordem, não-local }}^{\mid}$Similaridade MM5 & Noah LSM \\
\hline
\end{tabular}



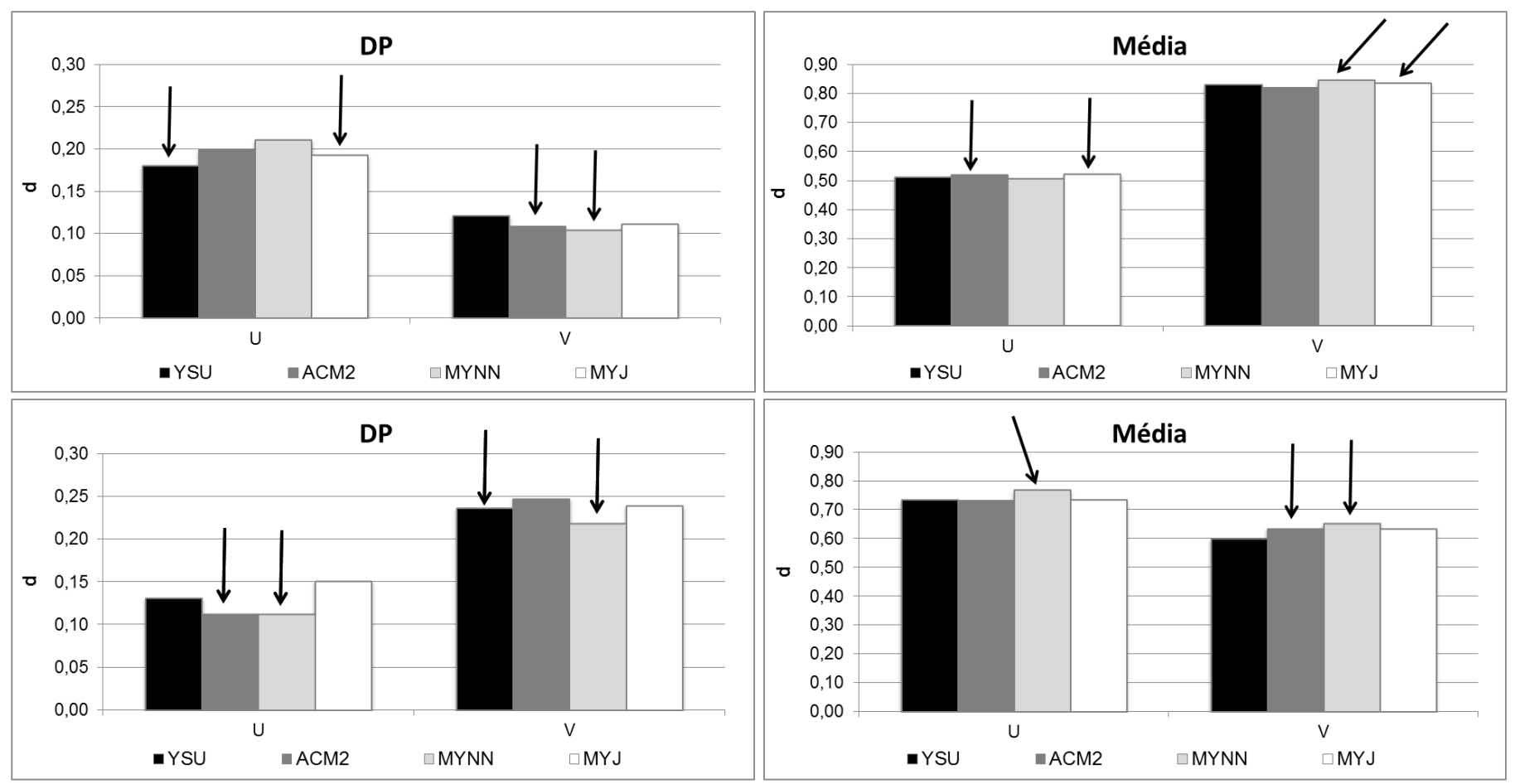

Figura 4 - Desvios-padrão (DP) e médias do índice de concordância de Willmott (d) das simulações de 72 horas com as quatro diferentes parametrizações de CLP para U e V para 2008 (paineis superiores) e 2010 (paineis inferiores). As setas indicam as mais altas médias e os mais baixos desvios-padrão.

si nesta figura, verificou-se que para o período chuvoso, a parametrização que apresentou índices melhores que as demais em maior número de vezes foi a MYNN2,5, seguida da ACM2. Entretanto, para o período seco, os resultados não se mostraram bem definidos.

Em conjunto, estes resultados levaram ao entendimento de que quando há nuvens com precipitação ao redor, existem modelos de parametrização que se adaptam melhor à microfísica local. Por outro lado, durante a época seca, embora haja nebulosidade (não precipitante), os diferentes esquemas funcionam de forma muito semelhante, não existindo uma parametrização específica que se mostre superior às demais.

Desta forma, as parametrizações ACM2 e MYNN2,5 foram os esquemas de CLP que apresentaram os melhores resultados. As diferenças foram pequenas, da ordem da primeira à terceira casa decimal. Foi realizada, então, uma avaliação teórica dos vários tipos de parametrizações. Em resumo, o esquema $\mathrm{ACM} 2$, ao adicionar uma componente de difusão de vórtices ao transporte não-local, representa melhor a forma de perfis verticais, especialmente do vento, por causa dos fluxos de massa locais e não-locais serem definidos explicitamente. Contudo, este modelo de difusão de vórtices resulta em uma maior altura da CLP. A parametrização ACM2 é ainda adequada para o transporte consistente de qualquer quantidade atmosférica $(U, V, \theta)$ na $C L P$, mas mostra-se não eficiente na estação chuvosa, podendo originar uma cobertura nebulosa excessiva no topo da CLP (Carvalho, 2009). Por outro lado, o esquema MYNN2,5 mostra-se mais adequado para a estação chuvosa, especialmente em situações de presença de água na CLP e em nevoeiros. Corrige o crescimento da camada limite e o conteúdo de água líquida, trata físicas de condensação na CLP considerando $\theta_{1}$ e $\mathrm{q}_{\mathrm{w}}$

Desta forma, ficou definida a utilização do esquema ACM2 para as simulações do período seco e o esquema MYNN2,5 para o período chuvoso.

\subsection{Previsões de 72 Horas}

Tendo escolhido o padrão de configurações físicas a partir dos testes de sensibilidade, foram realizadas todas as simulações de previsão de 72 horas. As Figuras 5 e 6 mostram as séries temporais do índice de Willmott e das variáveis $\mathrm{U}, \mathrm{V}$, Vel e dir (direção) médias na camada até $5000 \mathrm{~m}$ para as quatro simulações de cada estação que apresentaram os melhores índices, definidas a partir de seus valores representativos (média e DP de Willmott).

Para o ano de 2008 (Figura 5), notou-se que os valores de $\mathbf{d}$ para a componente $\mathrm{V}$ foram todos superiores aos resultados da componente $\mathrm{U}$ e com uma maior constância ao longo do tempo: oscilaram ao redor de 0,90 , com um valor mínimo de 
A

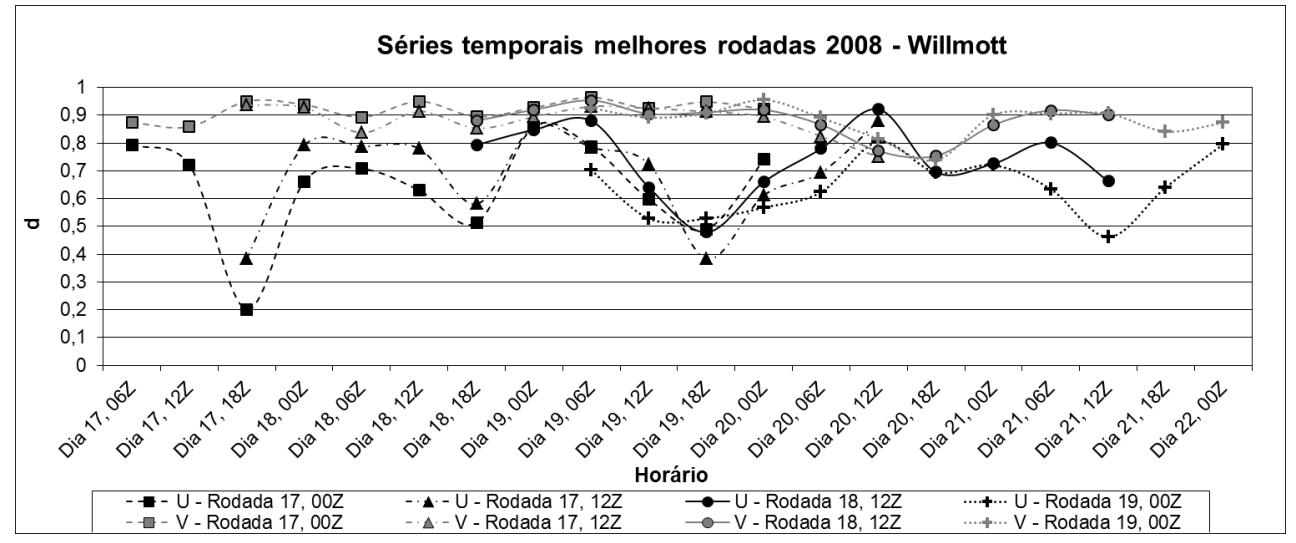

B

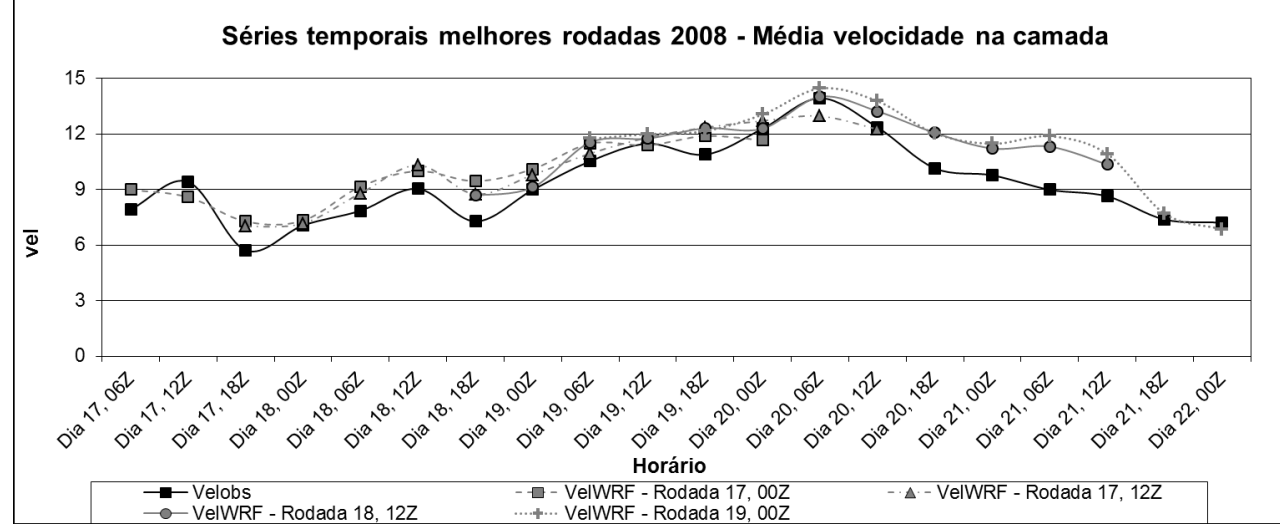

C

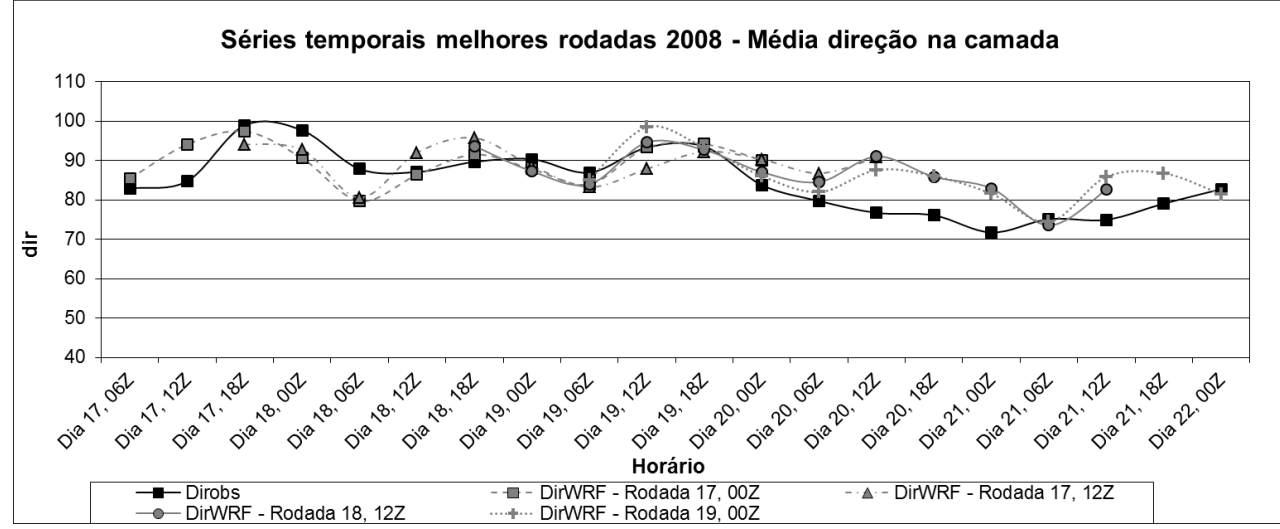

Figura 5 - Séries temporais de Willmott (a) e da velocidade e direção do vento médios na camada até 5000 m (b) e (c), observado e simulados, para as simulações selecionadas de 2008. Em (b) e (c), a linha preta contínua corresponde aos valores observados.

0,75 . Os valores para a componente U oscilaram entre $0,20 \mathrm{e}$ 0,90 . Também foram notáveis as fortes quedas em U sempre no período diurno (12 ou 18 UTC). Para a velocidade (não mostrado), a série apresentou valores mais altos, com variação entre 0,50 a 0,90, aproximadamente. Ainda com relação à estação seca, foram observados nos gráficos de valores médios da camada que, no geral, as simulações representaram bem os valores observados. Exceção apenas para a velocidade, superestimada pelo WRF em até $2,0 \mathrm{~m} / \mathrm{s}$, com média de 0,85 $\mathrm{m} / \mathrm{s}$ entre os desvios positivos e negativos.
Para o ano de 2010 (Figura 6), não houve superioridade entre $\mathrm{U}$ ou V. Os valores de d para U oscilaram de 0,45 a 0,95 e, para V, de 0,30 a 0,95. Para a velocidade, os valores também ficaram nesta faixa $(0,40$ a 0,90$)$. Na direção média da camada (6b), algumas simulações divergiram razoavelmente da observação. A componente U (6c) apresentou valores superestimados pelo WRF em até $3,0 \mathrm{~m} / \mathrm{s}$, sendo a média de $0,7 \mathrm{~m} / \mathrm{s}$, a mesma obtida para a velocidade total. As curvas de V (6d) mostraram-se satisfatórias, acompanhando bem a observação. 
A

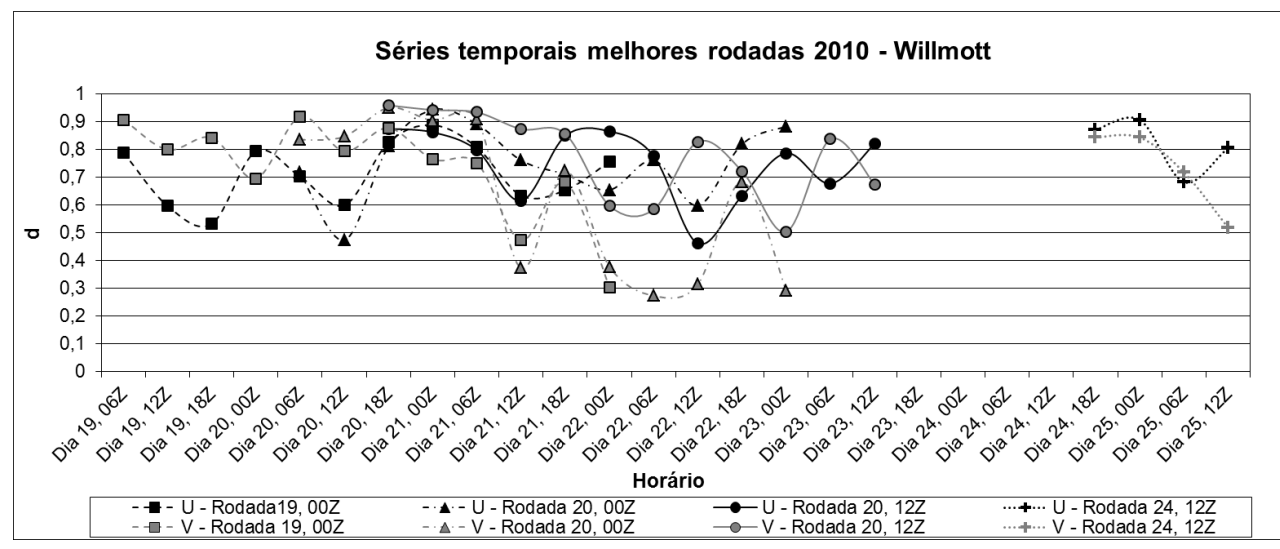

B

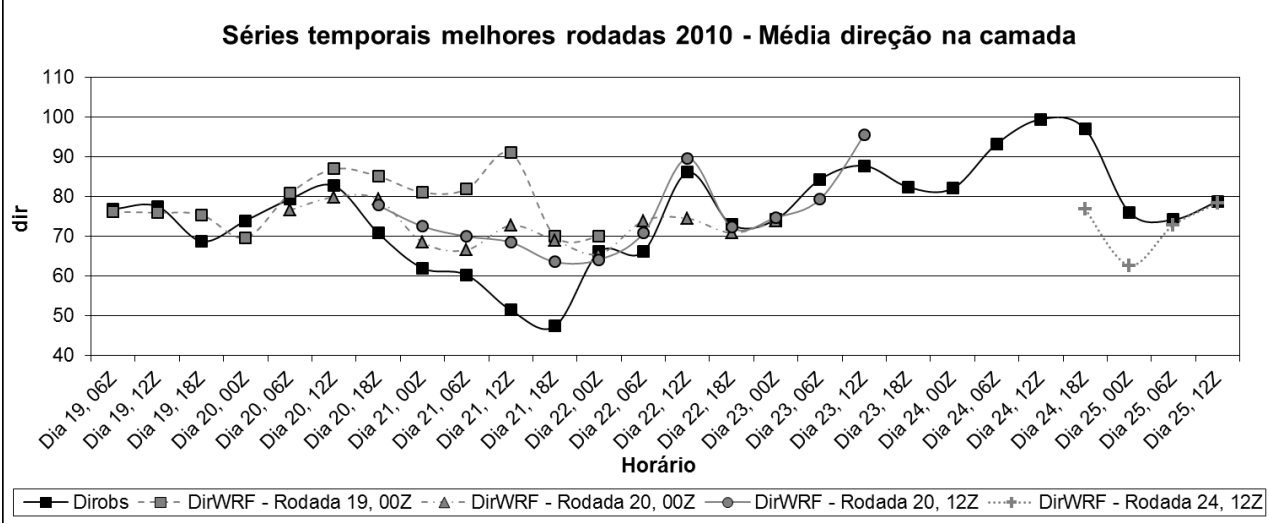

C

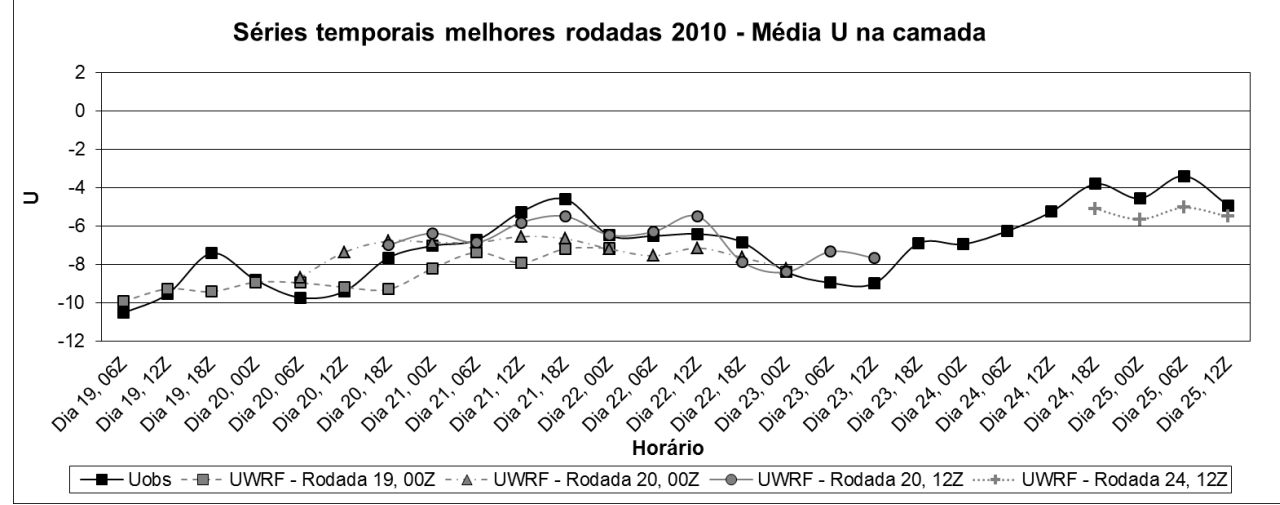

D

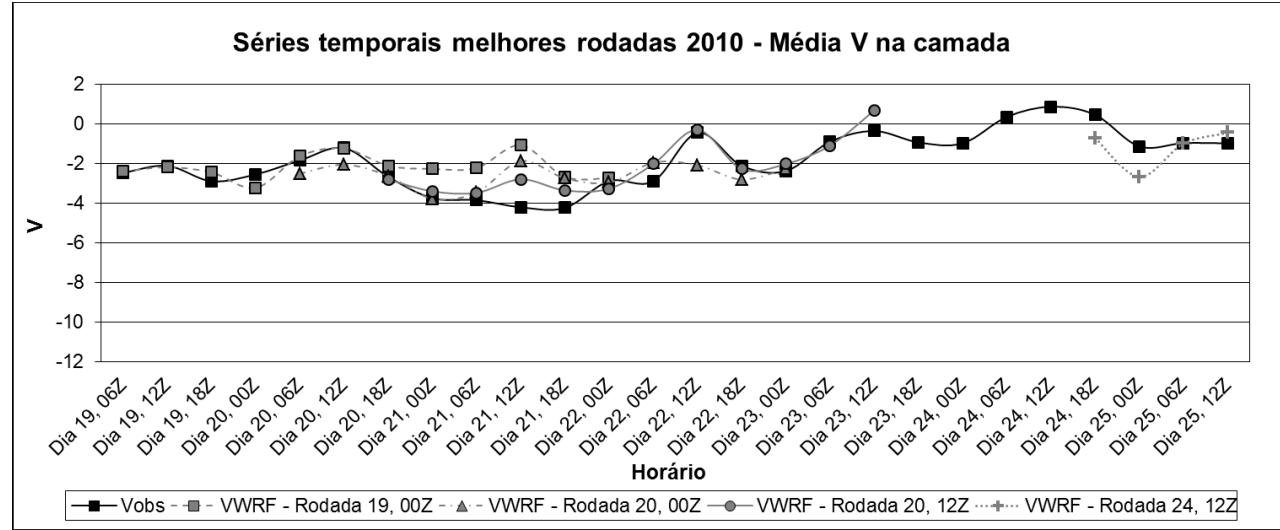

Figura 6 - Séries temporais de Willmott (a), da direção (b) e componentes U e V do vento (c) e (d) médios na camada até 5000 m, observado e simulados para as simulações selecionadas de 2010. 


\subsection{Coerência Termodinâmica}

As figuras de precipitação acumulada em 1 hora $(\mathrm{mm} / \mathrm{h})$ geradas pelo modelo WRF foram comparadas com imagens de intensidade de precipitação produzidas pela varredura de radar meteorológico no modo PPI (dBz). No geral, o modelo WRF não conseguiu posicionar corretamente os núcleos de chuva tanto no espaço quanto no tempo, assim como, também não foi eficiente em representar as intensidades de precipitação. Foram verificadas diversas defasagens de tempo, de 1 a 2 horas ou mais. Quando ocorreu o posicionamento correto de núcleos de chuva, sua intensidade foi sempre muito subestimada pelo modelo. Devido à metodologia utilizada, não foi possível quantificar com precisão a quantidade de chuva subestimada.

Um exemplo que merece destaque foi a forte precipitação observada pelo radar sobre o CLA durante 15 horas praticamente ininterruptas, das 16 UTC do dia 21 às 07 UTC do dia 22 de março de 2010, com núcleos de até $60 \mathrm{dBz}$, equivalente a aproximadamente $100 \mathrm{~mm} / \mathrm{h}$. Nenhuma das simulações que incluíram este intervalo temporal foi capaz de representar tal evento extremo. Somente após o cessar das chuvas observadas o modelo começou a representar fortes núcleos aos arredores do CLA, mas representando no máximo $30 \mathrm{~mm} / \mathrm{h}$ e deslocados, já que o núcleo principal foi observado sobre o CLA(Figura 7). É possível que o modelo global (GFS) tenha carregado este atraso em seus arquivos de previsão, utilizados como dados de entrada.

A Figura 8a mostra o campo das 13 UTC do dia 22 de março. Analisando as imagens de radar em horários próximos, tanto anteriores quanto posteriores, percebeu-se que os núcleos se assemelhavam com as observações do sistema que havia passado pela região de Alcântara entre 10 e 12 UTC. Ou seja, o modelo WRF representou a chuva com atraso de aproximadamente 2 horas. Isto pode ser um indicativo de que as nuvens estavam se deslocando mais rapidamente do que calculado pelo modelo WRF, mostrando uma defasagem temporal.

\section{CONCLUSÕES}

As diferenças entre as quatro parametrizações físicas de CLP testadas foram pequenas, com índices variando entre a primeira e a segunda casa decimal. Os esquemas que melhor se adequaram às características regionais e às condições sazonais
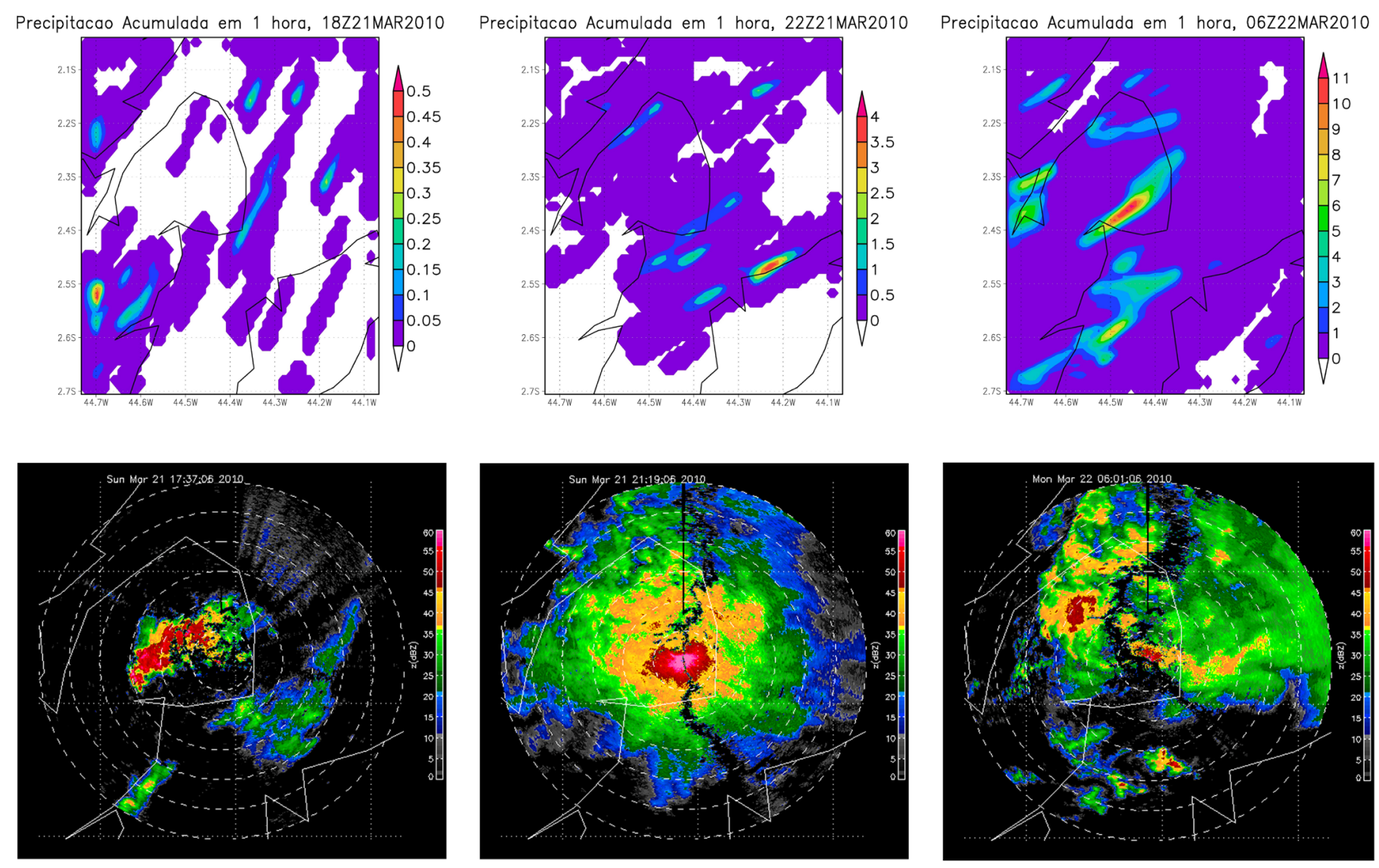

Figura 7 - Campos de precipitação acumulada (WRF) (campos superiores) e imagens de radar em raio 30 km (imagens inferiores). Forte núcleo de precipitação observado sobre o CLA. 
(a)

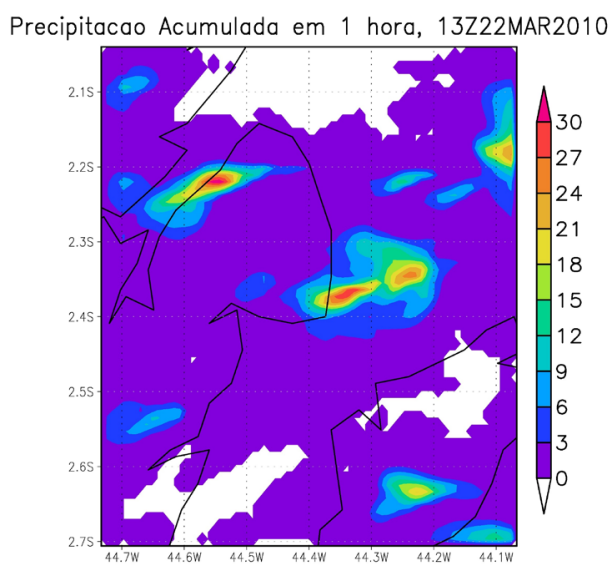

(b)

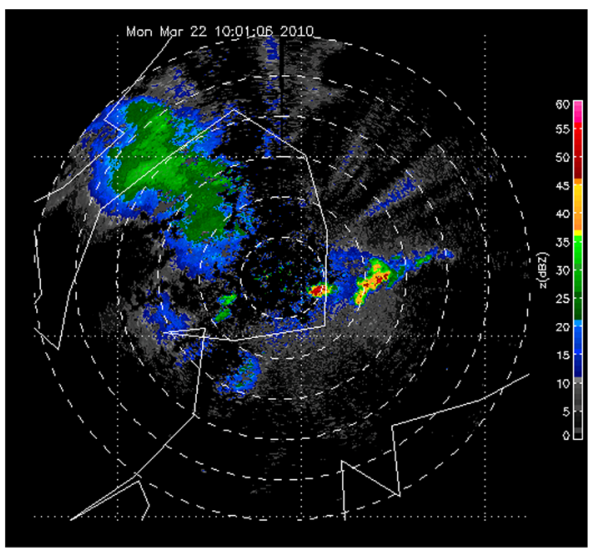

(c)

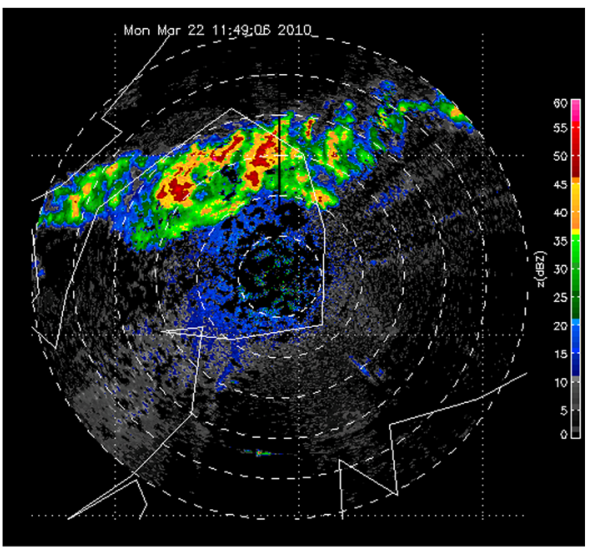

Figura 8 - Evolução do sistema convectivo: atraso temporal na simulação iniciada às 00 UTC do dia 22 de março. Precipitação acumulada de 12 às 13 UTC estimada pelo WRF (a) e intensidade de precipitação observada pelo radar às 10:01 UTC (b) e 11:49 UTC (c).

do clima de Alcântara foram: ACM2 para a estação seca e MYNN2,5 para a estação chuvosa. Entretanto, estas diferenças são pequenas de modo que, caso seja necessário para simulações operacionais, é possível realizar simulações com o modelo WRF com qualquer uma das parametrizações (ACM2 ou MYNN2,5) sem prejuízos.

O modelo WRF conseguiu representar o perfil do vento no CLA de forma razoável, dentro de suas limitações. Os valores alcançados pelo índice de Willmott foram considerados satisfatórios, de acordo com limites encontrados em outros estudos. Porém, não foi possível concluir que, depois de passado certo tempo de integração numérica (em intervalo de horas), as simulações tivessem apresentado um mesmo tipo de comportamento padrão em termos de melhora ou piora da qualidade de previsão.

Não foi possível identificar uma melhor performance para a estação seca ou chuvosa. No entanto, em termos das métricas utilizadas, as componentes do vento ( $\mathrm{U}$ e V) foram melhor representadas na estação chuvosa. Considerando o vetor velocidade do vento (calculado a partir das componentes do vento), a estação seca apresentou melhores resultados. Da mesma forma, considerando os valores médios na camada até 5000 m (que são utilizados para cálculo de ventos balísticos, usados na determinação da trajetória dos foguetes de sondagem), as simulações da estação seca se aproximaram mais das observações. Em geral, o modelo superestimou a velocidade do vento médio observado na camada em até $2,0 \mathrm{~m} / \mathrm{s}$, com média de $0,85 \mathrm{~m} / \mathrm{s}$ na estação seca e $0,70 \mathrm{~m} / \mathrm{s}$ na estação chuvosa. Acredita-se que este seja um resultado positivo no sentido de que, dentre as opções de parametrizações da CLP disponíveis, ambas as escolhidas se mostraram eficientes para os objetivos a que foram definidas, ou seja, representar a distribuição de momentum local de acordo com as características da estação.

O modelo WRF, com a configuração default de microfísica de nuvens, não consegue capturar a presença de chuva em termos de posicionamento e intensidade. Este tema deve ser ainda melhor estudado em trabalhos futuros.

\section{AGRADECIMENTOS}

Os autores agradecem à CAPES pela concessão da bolsa de Mestrado e ao CNPq [processos números 559949/2010-3 e 303720/2010-7 (bolsa PQ)]. Agradecem-se ainda a Jorge Yamasaki e Paulo Iriart pela ajuda e suportes prestados durante a execução deste trabalho.

\section{REFERÊNCIAS BIBLIOGRÁFICAS}

AGÊNCIA ESPACIAL BRASILEIRA (AEB). Plano Nacional de Atividades Espaciais (PNAE) 2012-2021. Disponível em: <www.aeb.gov.br>. Acesso em: 08 fev. 2013.

CARVALHO, D. J. S. Optimização do modelo numérico de previsão do tempo WRF no contexto de previsão e produção de energia eólica. 2009. 60 p. Dissertação (Mestrado em Meteorologia e Oceanografia Física) Universidade de Aveiro, Aveiro, Portugal, 2009.

CARVALHO, D.; ROCHA, A.; GÓMEZ-GESTEIRA, M.; SANTOS, C. A sensitivity study of the WRF model in wind simulation for an area of high wind energy. Environmental Modelling \& Software, v. 33, p. 23-34, 2012.

COUTINHO, E. C.; FISCH, G. Distúrbios Ondulatório de Leste (DOL's) na região do Centro de Lançamento de Alcântara 
- MA. Revista Brasileira de Meteorologia, v. 22, n. 2, p. 193-203, 2007.

DAVIS, C.; WANG, W.; DUDHIA, J. Does increased horizontal resolution improve hurricane wind forecasts? Weather and Forecasting, v. 25, n. 6, p. 1826-1841, 2010.

EMERY, C.; TAI, E.; YARWOOD, G. Enhanced meteorological modeling and performance evaluation for two Texas ozone episodes. Austin, TX: Environment International Corporation, 2001.

FISCH, G. Características do perfil vertical do vento no Centro de Lançamento de foguetes de Alcântara (CLA). Revista Brasileira de Meteorologia, v. 14, n. 1, p. 11-21, 1999.

GISLER, C. A. F. Análise do perfil de vento na camada limite superficial e sistemas meteorológicos atuantes no Centro de Lançamento de Alcantâra. 2009. 143 p. (INPE16079-TDI/1536). Dissertação (Mestrado em Meteorologia) - Instituto Nacional de Pesquisas Espaciais, São José dos Campos, 2009. Disponível em: <http:/urlib.net/sid.inpe.br/ mtc-m18@80/2009/04.24.12.33>.Acesso em: 31 out. 2011.

IAE/ACA; ICEA/DECEA. Relatório Experimento Chuva GPM 2010. São José dos Campos, SP, 2010. 26 p. Acesso em: 05 mar. 2012.

MARCIOTTO, E. R.; FISCH, G.; MEDEIROS, L. E. Characterization of surface level wind in the Centro de Lançamento de Alcântara for use in rocket structure loading and dispersion studies. Journal of Aerospace Technology and Management, v. 4, n. 1, p. 69 - 79, 2012.

NOLAN, D. S.; ZHANG, J. A.; STERN, D. P. Evaluation of planetary boundary layer parameterizations in tropical cyclones by comparison of in situ observations and highresolution simulations of hurricane Isabel (2003). Part I: initialization, maximum winds, and the outer-core boundary layer. Monthly Weather Review, v. 137, n.11, p. 36513674, 2009.
PIRES, L. B. M. Estudo da camada limite interna desenvolvida em falésias com aplicação para o Centro de Lançamento de Alcântara. 2009. 165 p. (INPE-16566TDI/1562). Tese (Doutorado em Meteorologia) - Instituto Nacional de Pesquisas Espaciais, São José dos Campos, 2009. Disponível em: <http://urlib.net/sid.inpe.br/mtcm18@80/2009/06.22.18.27>. Acesso em: 31 out. 2011.

ROBALLO, S. T.; FISCH, G. Escoamento Atmosférico no Centro de Lançamento de Alcântara (CLA): Parte I - Aspectos Observacionais. Revista Brasileira de Meteorologia, v. 23, n. 4, p. 510-519, 2008.

SILVA, A. F. G. Avaliação do modelo WRF ao perfil do vento no Centro de Lançamento de Alcântara. 2013. 98 p. (sid.inpe.br/mtc-m19/2013/02.07.16.21-TDI). Dissertação (Mestrado em Meteorologia) - Instituto Nacional de Pesquisas Espaciais (INPE), São José dos Campos, 2013. Disponível em: <http://urlib.net/8JMKD3MGP7W/3DGJ992>. Acesso em: 09 ago. 2013.

SKAMAROCK, W. C.; KLEMP, J. B.; DUDHIA, J. et al. NCAR Technical Note: A description of the Advanced Research WRF version 3. [S.1: s.n.], 2008, 113 p.

WANG, C.; HU, J.; FENG, S.; JIN, S.; ZHANG, F.; LIU, C. Comparing different boundary layer schemes of WRF by simulation the low-level wind over complex terrain. In: International Conference on Artificial Inteligence, Management Science and Electronic Commerce, 2., 2011, Zhengzhou, China. Proceedings... Zhengzhou: AIMSEC, 2011. p. 6183-6188.

WILlMOTT, C. J. On the validation of models. Physical Geography, v. 2, n. 2, p. 184-194, 1981. 